\title{
Coupon Collector's Test for Random Digits
}

1. Introduction. Increasing use of random numbers, especially in Monte Carlo procedures and in large computing installations, has served to focus attention on the various tests for randomness. Kendall and BABIngTon-Smith ${ }^{1}$ list four tests for so-called local randomness. While not giving the coupon collector's test (to be described below) a place in their now classical list of four tests, they did use a modified coupon collector's test in some of their investigations.

In an ordered set of digits, say, one may count the length of a sequence, beginning at a specified position, necessary to give or include the complete set of all ten digits. Or one may count the length required to give a set of $k, k<10$, different digits. The distribution of these observed lengths for different initial positions can then be compared with a theoretically computed distribution. Such a test will be called the coupon collector's test from an analogy with certain sales promotion schemes.

The theoretical distribution may be computed from formulas given by $\mathrm{H}$. von Schelling, ${ }^{2}$ which formulas hold for the case where the individual category probabilities might be unequal. For the random digital case with category probabilities equal to $1 / 10$, von Schelling's formulas simplify readily and may be conveniently related to the "differences of zero." These latter quantities are tabulated by FISHER and YATES ${ }^{3}$ up to sequences of length 26. But in using the coupon collector's test for a complete set of all 10 digits it has been found that the mean of the length distribution is slightly greater than 29 , and a table of probabilities associated with the sequence lengths 10 to 26 inclusive would hardly give a realistic picture of the entire distribution.

The present author has therefore extended this tabulation, and exact probabilities are given for sequence lengths $10 \leq n \leq 35$ and approximate probabilities for sequence lengths $36 \leq n \leq 75$. The probabilities were computed from the relation

$$
p_{n}=\frac{1}{10^{n-1}} \sum_{j=0}^{q}(-1)^{j}\left(\begin{array}{c}
q \\
j
\end{array}\right)(q-j)^{n-1},
$$

and are listed in Table 3.

If one were interested in sequence lengths necessary to obtain five different digits, the mean of this distribution is approximately 6.46 . The range 5 to 26 inclusive available from FISHER and YATES ${ }^{3}$ might be sufficient here.

2. Sequence Lengths for Decimal Expansions of $\pi$ and $e$. It would be a simple matter to program a large digital computing machine so that it would tabulate the distribution of the sequence lengths needed for complete sets for a given ordered digital collection. However, the author did not have such a digital computing machine available, and he made a tabulation by hand for the decimal expansion of $\pi$. The 2035 decimal approximation to $\pi$ given by GEORGE W. REITWIESNER $^{4}$ was used as the raw material for this count. Beginning with the initial position 3 in $\pi \simeq 3.14159 \cdots$, it was recorded that a sequence length of 33 
positions was needed to get all the ten digits. Beginning anew with the thirtyfourth position digit (which is a 2), it was recorded that a sequence of 18 positions was needed to get a complete set of all the 10 digits. Continuing this procedure, 67 sequences of complete sets were obtained, plus an incomplete sequence (at the end of the decimal expansion) of length 15. It was considered advisable to make the sequences non-overlapping as described above since there is considerable dependence among the set of sequence lengths if every position in the decimal expansion of $\pi$ is regarded as a new starting point.

The sequence lengths for $\pi$ are also included in Table 3. This tabulation for $\pi$ was checked by Mr. Wayne Jones of the Department of Defense, Washington, D. C.

Mr. Jones also made a tabulation based on the decimal expansion of $e$. REITWIESNER ${ }^{4}$ gave a 2010 decimal approximation to $e$. An additional 490 places was given by Metropolis, Reitwiesner and von Neumann. ${ }^{5} \mathrm{Mr}$. Jones found 82 complete sequences using 2486 digits in the expansion of $e$. This tabulation is also given in Table 3. The author desires to thank Mr. Jones for this count.

3. Statistical Tests. The mean and the standard deviation of the theoretical distribution may be computed from results given by von ScHELLING ${ }^{2}$ or FELLER. ${ }^{6}$ These theoretical values and the corresponding observed values for $\pi$ and $e$ are given below.

\begin{tabular}{|c|c|c|c|}
\hline \multicolumn{4}{|c|}{ TABLE 1} \\
\hline & Theoretical & $\pi$ & $e$ \\
\hline $\begin{array}{l}\text { Mean } \\
\text { Standard deviation }\end{array}$ & $\begin{array}{l}29.29 \\
11.21\end{array}$ & $\begin{array}{l}30.16 \\
11.83\end{array}$ & $\begin{array}{l}30.32 \\
10.64\end{array}$ \\
\hline
\end{tabular}

To use a chi-square test, it is desirable that the expected values all exceed 10 in size. Since the sample size for $\pi$ is small (67) some grouping of the sequence lengths is necessary to meet this desired minimum. The following results were obtained for a convenient grouping.

TABLE 2

\begin{tabular}{lcccc} 
& \multicolumn{2}{c}{$\pi$} & \multicolumn{2}{c}{$e$} \\
Sequence lengths, $n$ & Observed & Expected & Observed & Expected \\
$10-19$ & 13 & 11.604 & 12 & 14.202 \\
$20-23$ & 13 & 11.720 & 11 & 14.344 \\
$24-27$ & 9 & 11.491 & 14 & 14.064 \\
$28-32$ & 5 & 11.480 & 15 & 14.050 \\
$33-39$ & 13 & 10.195 & 17 & 12.477 \\
40 and over & 14 & 10.510 & 13 & 12.863 \\
Totals & 67 & 67.000 & 82 & 82.000 \\
Chi-squared test & \multicolumn{2}{c}{6.436} & & \multicolumn{2}{c}{2.826} \\
$\quad$ values & \multicolumn{2}{c}{}
\end{tabular}

Neither of these chi-square test values is unusually out of line. It has been previously reported ${ }^{5,7}$ that (using a sample of 2000 digits for $e$ ) excessive flatness in the single frequencies was noted, and an indication was obtained that the single digits in $e$ are "non-random." Apparently, this phenomenon did not reflect itself 
TABLE 3

Table of Probabilities and Empirical Distributions

Observed for

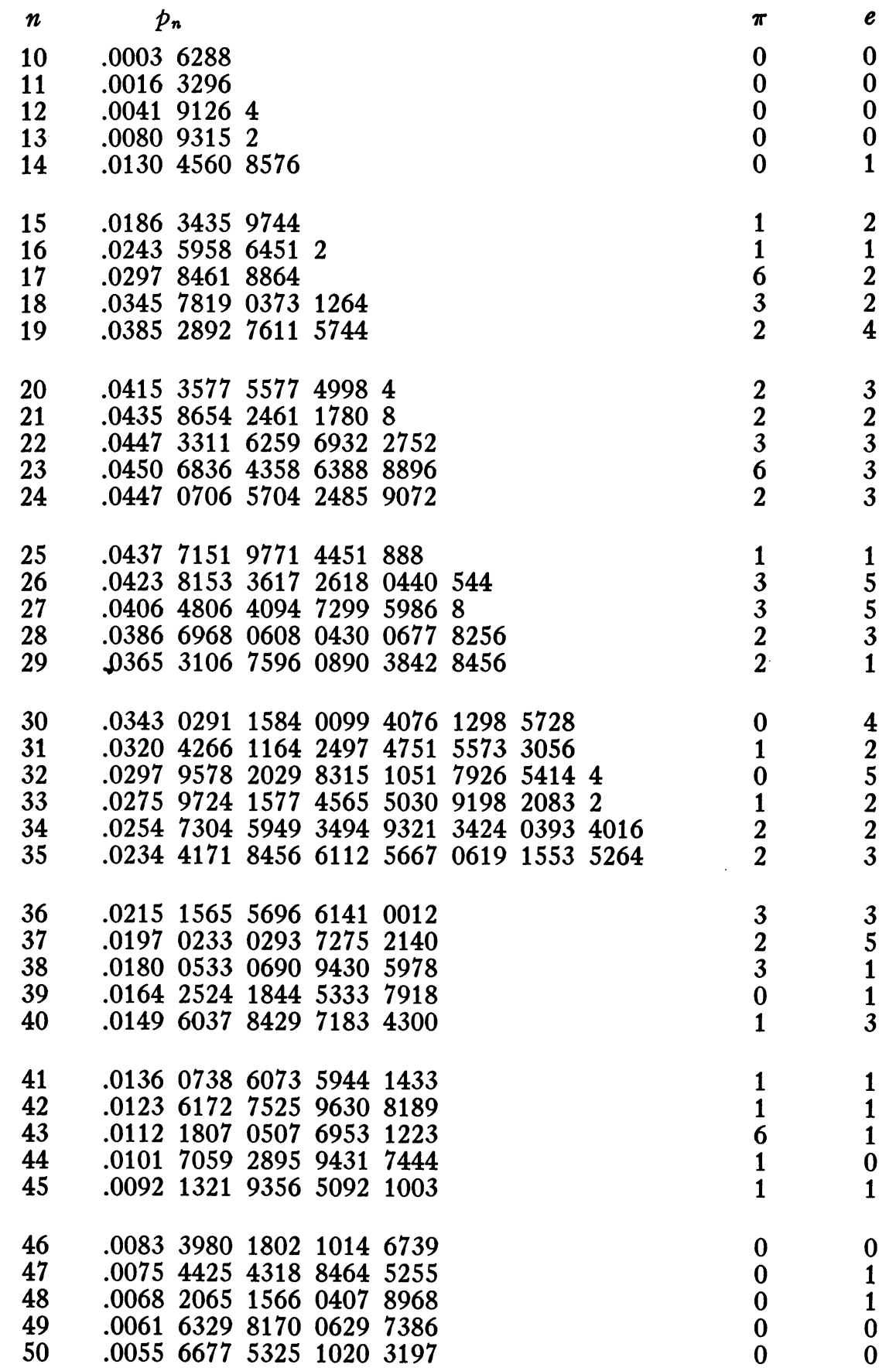




\section{TABLE 3-Continued}

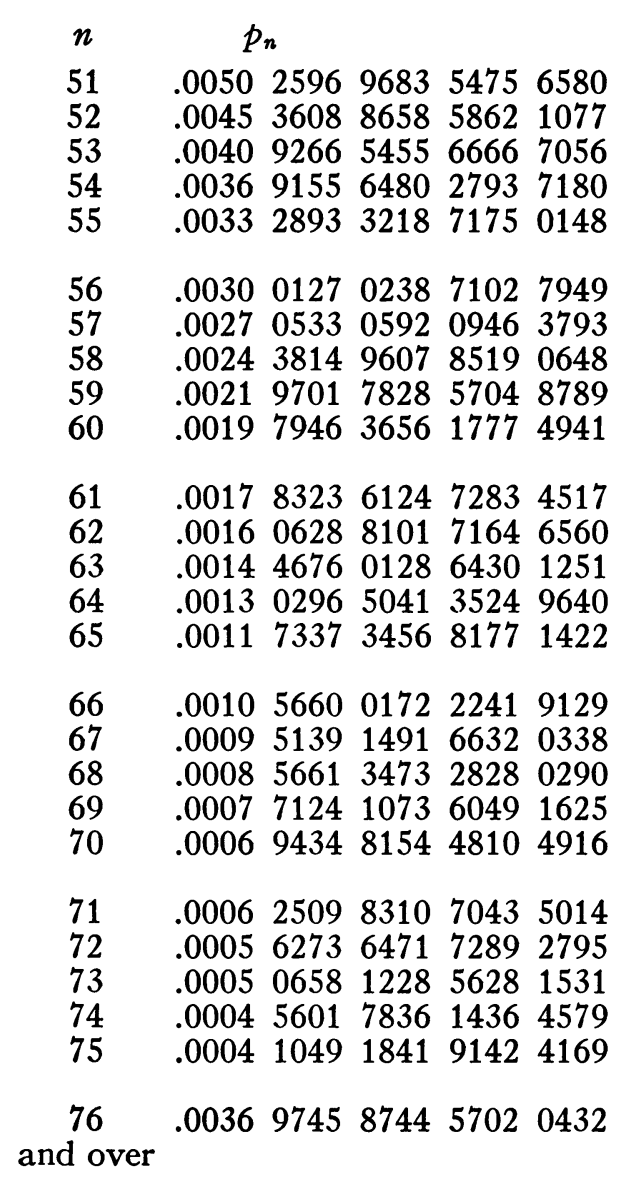

Observed for

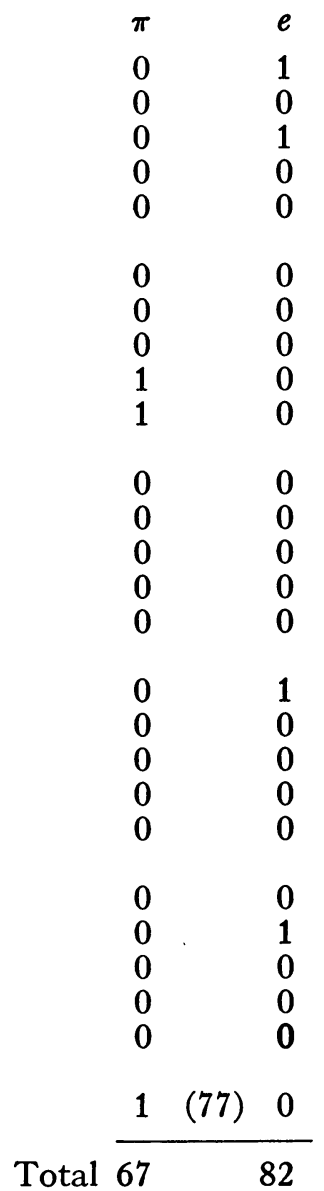

in materially changing the characteristics of the sequence length distribution for the coupon collector's test. Some question arises as to whether the single frequency test and the coupon collector's test are independent, and also which test has the greater power.

The chi-square test values in Table 2 were calculated by assuming that the sequence lengths for complete sets of digits are independent draws from a known (infinite) multinomial probability distribution. (Null hypothesis.) The alternatives would include unspecified sorts of dependency and other underlving probabilities different from those given in Table 3.

The University of Texas

Robert E. Greenwood

Austin, Texas

${ }^{1}$ M. G. Kendall \& B. Babington Smith, "Randomness and random sampling numbers," Royal Stat. Soc., Jn., v. 101, 1938, p. 147-166.

${ }^{2}$ H. von Schelling, "Auf der Spur des Zufalls," Deutsches Statisches Zentralblatt, v. 26, 1934, p. 137-146. Also, "Coupon collecting for unequal probabilities," Amer. Math. Mon., v. 61, 1954, p. 306-311. 
${ }^{3}$ R. A. Fisher \& F. YATEs, Statistical Tables for Biological Agriculture and Medical Research. 3rd edition, London, 1948, Table XXII.

4 GEORGE W. REITWIESNER, "An ENIAC determination of $\pi$ and $e$ to more than 2000 decimal places," $M T A C$, v. 4, 1950, p. 11-15.

s N. C. Metropolis, G. Reitwiesner, \& J. von Neumann, "Statistical treatment of values of first 2000 decimal digits of $e$ and $\pi$ calculated on the ENIAC," MTAC, v. 4, 1950, p. 109-111.

${ }^{\circ}$ W. Feller, Probability Theory and Its Applications. Volume 1, New York, 1950, p. 175-181.

${ }^{7} \mathrm{~F}$. GRUENBERGER, "Further statistics on the digits of $e, " M T A C, \mathrm{v} .6,1952, \mathrm{p}$. 123-134.

\section{A Method for the Evaluation of a System of Boolean Algebraic Equations}

With the advent of large scale electronic devices whose logical design is described by a system of Boolean algebraic equations, a method to mechanize the evaluation of such a system and shorten this evaluation with respect to time will be increasingly useful. Such a method will be described in this paper.

The problem may be described as follows: Given a set of $n$ variables, $Q^{k}$, $(k=1,2, \cdots, n)$ each of which may take on the value 1 (true) or 0 (false) at any time $t$; then the value of any $Q^{k}$ at time $t+1$ may be defined by the system of Boolean equations

$$
\begin{aligned}
R_{t}{ }^{k} & =f_{k}\left(Q_{t}{ }^{q}\right) \\
S_{t}{ }^{k} & =g_{k}\left(Q_{t^{q}}\right) \\
Q^{k}{ }_{t+1} & =\phi\left(Q_{t^{k}}{ }^{2} R_{t^{k}}{ }^{k} S_{t}{ }^{k}\right)
\end{aligned}
$$

where $1 \leq q \leq n$. For example, the recirculation loop of a dynamic flip-flop may be defined simply by

$$
Q^{k}{ }_{t+1}=\phi\left(Q_{t}{ }^{k}, R_{t}{ }^{k}, S_{t}{ }^{k}\right)=R_{t}^{k} .
$$

In another system, a more complex definition

$$
Q^{k}{ }_{t+1}=\phi\left(Q_{t}{ }^{k}, R_{t}{ }^{k}, S_{t}{ }^{k}\right)=Q_{t}{ }^{k} \cdot \overline{R_{t}{ }^{k}} \cdot \overline{S_{t}{ }^{k}}+R_{t}{ }^{k} \cdot \overline{S_{t}{ }^{k}}+\overline{Q_{t}{ }^{k}} \cdot R_{t}{ }^{k} \cdot S_{t}{ }^{k}
$$

may be taken, where $R_{t}{ }^{k}$ and $S_{t}{ }^{k}$ are the two inputs to flip-flop $Q^{k}$.

We shall use the symbols for conjunction, disjunction, and negation

$$
\begin{array}{lll}
Q^{1} \cdot Q^{2} & \text { " } Q \text { and } Q^{2 "} & \text { conjunction } \\
Q^{1}+Q^{2} & \text { " } Q \text { or } Q^{2 "} & \text { disjunction } \\
Q^{1} & \text { "Not } Q 1 " & \text { negation }
\end{array}
$$

which are defined by the truth tables ${ }^{1}$

$\begin{array}{ccccc}Q^{1} & Q^{2} & Q^{1} \cdot Q^{2} & Q^{1}+Q^{2} & \overline{Q^{1}} \\ 0 & 0 & 0 & 0 & 1 \\ 0 & 1 & 0 & 1 & 1 \\ 1 & 0 & 0 & 1 & 0 \\ 1 & 1 & 1 & 1 & 0 .\end{array}$

A "term" is defined as one or more variables conjoined together, e.g., $Q^{1} \cdot Q^{2} \cdot \overline{Q^{3}}$; and an "equation" as $M$ terms, $T_{m},(m=1,2, \cdots, M)$ disjoined together, e.g., $Q^{1} \cdot Q^{2} \cdot \overline{Q^{3}}+Q^{1} \cdot Q^{4}$. Now note that the value of a term is zero if any variable in 